\title{
TRAYECTORIA POLÍTICA EDUCATIVA DEL PROFESORADO DE PORTUGUÉS EN URUGUAY
}

\author{
TRAYECTÓRIA POLÍTICA EDUCACIONAL \\ DO PROFESSORADO DE PORTUGUÊS NO URUGUAI
}

\author{
EDUCATIONAL POLITICAL TRAJECTORY \\ OF PORTUGUESE TEACHER TRAINING IN URUGUAY
}

Edilson Teixeira ${ }^{1}$

\begin{abstract}
RESUMEN: Con el objetivo de explorar algunos aspectos del ciclo de políticas, con base en el enfoque teórico-metodológico propuesto por Ball $(1993,1998,2001)$, realizaremos un estudio documental sucinto para centrarnos en el proceso de construcción de políticas para la formación docente de portugués de la Administración Nacional de Educación Pública (ANEP) de Uruguay. Bowe, Ball y Gold (1992) aportaron conceptualmente los ciclos de políticas a las investigaciones sobre educación, a partir de dos interrogantes, ¿qué son las políticas educativas? y ¿cómo se puede investigar las políticas educativas, sus trayectorias y efectos? Sus trabajos constituyen una caja de herramientas para analizar el carácter complejo, conflictivo y multifacético del proceso de producción y puesta en acto de las políticas educativas. Ello permitió reconocer diferentes arenas donde se generan, delimitan y ponen en acto las políticas, al igual que sus relaciones y especificidades, denominadas como contexto de influencia, contexto de producción de los textos de las políticas, contexto de la práctica, contexto de los efectos y contexto de las estrategias políticas. En este artículo, haremos referencia a los componentes del ciclo de políticas y estudiaremos, en especial, algunos ejemplos iniciales de la trayectoria política. En el contexto de influencia abordaremos, brevemente, documentos de instituciones internacionales y regionales, con representantes de los Estados Nacionales, tales como la Declaración Universal Derechos Lingüísticos de 1996, de la UNESCO, y el Plan Estratégico de 2006-2010 del Sector Educativo del MERCOSUR. En el contexto de producción de los textos de las políticas nos detendremos en documentos de la Comisión de Políticas Lingüísticas para la Educación Pública de la ANEP, la Ley General de Educación y el Plan de Estudio del Profesorado de Portugués de 2008, porque permitieron la puesta en acto del actual profesorado de portugués en la educación pública uruguaya.
\end{abstract}

Palabras-clave: Políticas públicas. Educación uruguaya. Profesorado de portugués. Ciclo de políticas.

RESUMO: Com o objetivo de explorar alguns aspectos dos ciclos de política, com base na abordagem teórico-metodológica proposta por Ball $(1993,1998,2001)$, faremos um estudo documental sucinto com ênfase no processo de construção de políticas para a formação docente de portugueses da Administração Nacional de Educação Pública (ANEP) do Uruguai. Bowe, Ball e Gold (1992) contribuíram conceitualmente com os ciclos de políticas para as pesquisas em educação, com base em duas questões, o que são políticas educacionais? Como podem ser investigadas as políticas educacionais, suas trajetórias e efeitos? Seus trabalhos constituem uma caixa de ferramentas para analisar a natureza

\footnotetext{
${ }^{1}$ Profesor de Portugués, Español y Literatura (ANEP, Uruguay). Licenciado en Investigación Lingüística (UDELAR, Uruguay). Realizó cursos de posgrados como "Diploma de Especialización en la enseñanzaaprendizaje de Portugués LE" (UDELAR, USP y UFRGS), "Diploma Métodos y Materiales de Enseñanza de Español LE" (Fundación Universitaria Iberoamericana, Universidad de León, España), "Curso de EspañolPortugués para el Intercambio-CEPI" (UNC, UNER, UFRGS y UDELAR) y "Formación en acción, integración de las tecnologías en la formación docente" (FLACSO, CFE, Fundación Telefónica-Movistar). Acredita cursos de Maestría en Ciencias Humanas "Lenguaje, cultura y sociedad" (UDELAR) y del Master Sciences du Langage spécialité Français Langue Etrangère (Université Stendhal Grenoble III, Francia). Actualmente, cursa el Doctorado en Educación (UNR-FHYA, Argentina). ORCID: https://orcid.org/0000-0002-6213-6371. E-mail: edilson.uy@interbraslinguas.com.
} 
complexa, conflitiva e multifacetada do processo de produção e prática das políticas educacionais. Isso possibilitou reconhecer diferentes arenas onde as políticas são geradas, delimitadas e colocadas em ação, bem como suas relações e especificidades, chamadas de contexto de influência, contexto de produção de textos das políticas, contexto da prática, contexto dos efeitos e contexto das estratégias políticas. Neste artigo, faremos referência aos componentes do ciclo de políticas e estudaremos, em particular, alguns exemplos iniciais da trajetória política. No contexto de influência, analisaremos brevemente documentos de instituições internacionais e regionais, com representantes dos Estados Nacionais, como a Declaração Universal dos Direitos Linguísticos de 1996, da UNESCO, e o Plano Estratégico 20062010 do Setor Educacional do MERCOSUL. No contexto da produção dos textos das políticas, examinaremos documentos da Comissão de Políticas de Línguas para a Educação Pública da ANEP, da Lei Geral de Educação e o Plano de Estudos do Professorado de Português de 2008, porque permitiram pôr em ato o atual professorado de português na educação pública uruguaia.

Palavras-chave: Políticas públicas. Educação uruguaia. Professorado de português. Ciclo de políticas.

ABSTRACT: With the objective of exploring some aspects of the policy cycle, based on the theoreticalmethodological approach proposed by Ball $(1993,1998,2001)$, we will carry out a succinct documentary study to focus on the process of policy construction for Portuguese teacher training of the National Administration of Public Education (ANEP) of Uruguay. Bowe, Ball, and Gold (1992) conceptually contributed the policy cycles to research on education, based on two questions, what are educational policies? And how can educational policies, their trajectories, and effects be investigated? His work constitutes a toolbox to analyze the complex, conflictive, and multifaceted nature of the process of production and put in act of educational policies. This made it possible to recognize different arenas where policies are generated, delimited, and put into action, as well as their relationships and specificities, called the context of influence, the production's context of policy texts, the context of practice, the context of effects, and context of political strategies. In this article, we will refer to the components of the policy cycle and we will study, in particular, some early examples of the political trajectory. In the context of influence, we will briefly address documents of international and regional institutions, with representatives of the National States, such as the Universal Declaration of Linguistic Rights of 1996, of UNESCO, and the Strategic Plan of 2006-2010 of the Education Sector of MERCOSUR. In the context of the production of the policies' texts, we will dwell on documents of the Language Policy Commission for Public Education of ANEP, the General Law of Education and the Study Plan for Portuguese Teachers of 2008, because they allowed the put in action of the current Portuguese teacher training in Uruguayan public education.

Keywords: Public policies. Uruguayan education. Portuguese teacher training. Policy cycle.

\section{INTRODUCCIÓN}

Desde hace algunas décadas, los Estados comenzaron a promover la educación desde la perspectiva de derechos, de este modo, las políticas lingüísticas se han manifestado significativamente en diferentes instancias de organismos internacionales y nacionales, principalmente para las agendas educativas públicas. A nivel global, regional y nacional las políticas educativas transitan por diversas influencias como podremos ver concisamente en el caso de nuestro estudio.

Con el objetivo de explorar algunos aspectos de los ciclos de política, con base en el enfoque teórico-metodológico propuesto por Stephen Ball, realizaremos un estudio documental sucinto y nos centraremos en el proceso de construcción de políticas para la formación docente de portugués de la Administración Nacional de Educación Pública (ANEP).

Bowe, Ball y Gold (1992) aportaron conceptualmente los ciclos de políticas a las investigaciones sobre educación, partiendo de dos interrogantes, ¿qué son las políticas educativas? y ¿cómo se puede investigar las políticas educativas, sus trayectorias y efectos? Sus 
trabajos constituyen una caja de herramientas para analizar el carácter complejo, conflictivo y multisituado del proceso de producción y puesta en acto de las políticas educativas. Ello permitió reconocer diferentes arenas donde se generan, delimitan y ponen en acto las políticas, al igual que sus relaciones y especificidades, denominadas como contexto de influencia, contexto de producción de los textos de las políticas, contexto de la práctica, contexto de los efectos y contexto de las estrategias políticas (BOWE, BALL y GOLD, 1992). Este abordaje teórico-metodológico busca, por un lado, superar las perspectivas lineales y simplistas de la implementación en las cuales las políticas son vistas como producción acabada del Estado, instituidas o no por las escuelas, y, por otro lado, enfatizar la complejidad de intereses e influencias al momento de definir las políticas educativas (BEECH y MEO, 2016).

Miranda (2011) expresa que las políticas están configuradas en una trama de contextos no lineales interrelacionados, con formas de interpretar el mundo y de cambiar el modo de pensar en lo que hacemos. Estos contextos se entienden como "arena de actividad social y discursiva a través de los cuales las políticas se mueven y, dentro de las cuales, las políticas son producidas, creadas y ejecutadas" (MIRANDA, 2011, p. 109). En tal sentido, Ball (2016) argumenta que el ciclo de políticas es un método de investigación y teorización sobre políticas $\mathrm{y}$, por lo tanto, propone un esquema flexible con herramientas sociológicas para dar sentido a la política. De este modo, es necesaria una perspectiva combinada para lidiar con la confusión de las realidades, propia de un proceso político.

En este artículo, si bien haremos referencia a varios componentes del ciclo de política, no desarrollaremos todos los contextos. Específicamente, estudiaremos algunos ejemplos iniciales de la trayectoria política; en el contexto de influencia abordaremos, brevemente, instituciones internacionales y regionales; en el contexto de producción de los textos de las políticas nos detendremos en producciones de documentos políticos que permitieron implementar la carrera de profesores de portugués en la educación pública uruguaya.

\section{CICLO DE LAS POLÍTICAS}

Inicialmente, los autores propusieron un ciclo continuo de tres contextos principales interrelacionados, sin una dimensión temporal o secuencial, los cuales desarrollaremos en este estudio. Además, Ball (1997) agregó dos contextos, que podrán ser trabajados en otra oportunidad. El cuarto contexto, de efectos generales y específicos, se ocupa de cuestiones de justicia, igualdad y libertad individual. El quinto contexto, de estrategia política, implica la identificación de un conjunto de actividades sociales y políticas necesarias para enfrentar desigualdades creadas o reproducidas por la política investigada.

\section{PRIMER CONTEXTO}

En el contexto de influencia (BALL, 1998) se inician las políticas públicas y se construyen los discursos. Además de presentarse los escenarios, los grupos interesados se disputan para influir en las definiciones y propósitos sociales de las políticas educativas.

Ball reconoce las influencias internacionales sobre el proceso de creación de políticas nacionales. Dichas influencias son reinterpretadas por los Estados-nación, en tal sentido se reconoce una interrelación dialéctica entre lo global y lo local. La globalización promueve la migración de políticas, sin ser una mera transposición, puesto que las políticas son recontextualizadas dentro de contextos nacionales específicos (ROBERTSON, 2003; BALL, 1998 y 2001).

Por esa razón, mencionaremos algunos documentos (declaraciones, recomendaciones, 
normativas) que, si bien son de organismos del ámbito supranacional, participan representantes de los Estados Nacionales. Entre los efectos de influencias veremos, como segundo ciclo, las posteriores políticas lingüísticas y educativas públicas de Uruguay textualizadas principalmente en el Plan de estudio de 2008 de la formación docente de portugués.

Para trabajar la primera categoría en el análisis de las políticas públicas en cuestión, como instancias previas a la producción de textos nacionales, tomamos referencias de organismos internacionales y regionales. En primera instancia, traemos a colación la Carta Europea de las Lenguas Regionales o Minoritarias, de 1992, dado que es un documento considerado avanzado en la defensa de derechos lingüísticos.

\begin{abstract}
En materia de lenguas regionales o minoritarias, en los territorios en los que se hablen dichas lenguas y según la situación de cada una de ellas, las partes basarán su política, su legislación y su práctica en los objetivos y principios siguientes: a) el reconocimiento de las lenguas regionales o minoritarias como expresión de la riqueza cultural; b) el respeto del área geográfica de cada lengua regional o minoritaria, actuando de tal suerte que las divisiones administrativas ya existentes o nuevas no sean un obstáculo para el fomento de dicha lengua regional o minoritaria; c) la necesidad de una acción resuelta de fomento de las lenguas regionales o minoritarias, con el fin de salvaguardarlas; (...) (CMCE, 1992, Art.7).
\end{abstract}

Cada uno de estos enunciados constituye fundamentos que influenciaron significativamente en la Declaración Universal Derechos Lingüísticos (DUDL) de 1996, realizada en Barcelona durante la Conferencia Mundial de Derechos Lingüísticos con la participación de importantes organizaciones y expertos de casi un centenar de países, como defensa de la diversidad lingüística, avalada por la UNESCO y aprobada por las Naciones Unidas. A modo de ejemplo, destacamos algunos artículos revolantes paras nuestra temática:

Artículo 1.2

Parte del principio de que los derechos lingüísticos son a la vez individuales y colectivos, y adopta como referente de la plenitud de los derechos lingüísticos el caso de una comunidad lingüística histórica en su espacio territorial, entendido éste no sólo como área geográfica donde vive esta comunidad, sino también como un espacio social y funcional imprescindible para el pleno desarrollo de la lengua.

Sección II - Educación

Artículo 23

1. La educación debe contribuir a fomentar la capacidad de autoexpresión lingüística y cultural de la comunidad lingüística del territorio donde es impartida.

2. La educación debe contribuir al mantenimiento y desarrollo de la lengua hablada por la comunidad lingüística del territorio donde es impartida.

3. La educación debe estar siempre al servicio de la diversidad lingüística y cultural, y las relaciones armoniosas entre diferentes comunidades lingüísticas de todo el mundo.

4. En el marco de los principios anteriores, todo el mundo tiene derecho a aprender cualquier lengua.

Artículo 24

Toda comunidad lingüística tiene derecho a decidir cuál debe ser el grado de presencia de su lengua, como lengua vehicular y como objeto de estudio, en todos los niveles de la educación dentro de su territorio: preescolar, primario, secundario, técnico y profesional, universitario y formación de adultos (UNESCO, 1996).

Los discursos expuestos en los artículos citados son imprescindibles para crear y sostener políticas que contemplen hablantes de todas las lenguas, así como los propios organismos y sus acciones de gran influencia internacional. De ese modo, desde una perspectiva 
asociativa, podremos identificar tendencias y paradigmas relativamente congruentes $\mathrm{o}$ divergentes en lo que Ball designa como red de políticas globales (AVELAR, 2016). En tal sentido, en nuestro continente destacamos el Mercado Común del Sur (MERCOSUR), porque, además de ser un bloque económico, establece lenguas oficiales y la correspondiente enseñanza para la integración regional ${ }^{2}$. El Sector Educativo del MERCOSUR (SEM) es el espacio de coordinación de las políticas educativas que reúne los países miembros y asociados desde 1991, cuando el Consejo del Mercado Común (CMC) creó la Reunión de Ministros de Educación del MERCOSUR (RME), para "establecer un programa de formación de docentes para la enseñanza de las lenguas oficiales del Mercosur" (RME, 1993, act. 1/93, art. 8).

Desde entonces, nuevos discursos para el reconocimiento y respeto de la diversidad lingüística causaron ciertas rupturas en las posiciones nacionalistas de afirmación homogénea. Entendemos que el MERCOSUR constituye una fuerte influencia para el cambio político del Estado uruguayo, puesto que incidió como organismo supranacional en asuntos políticos, legales, económicos, sociales y, particularmente, educativos y culturales mediante el SEM. Asi vemos en el primer objetivo del Plan Estratégico 2006-2010: "Contribuir a la integración regional acordando y ejecutando políticas educativas que promuevan una ciudadanía regional, una cultura de paz y el respeto a la democracia, a los derechos humanos y al medio ambiente" con los lineamientos "Fomento de programas culturales, lingüísticos y educativos que contribuyan a construir una identidad regional y fortalezcan las zonas de frontera" y "Promoción y difusión de los idiomas oficiales del MERCOSUR" (SEM, 2006, p. 9).

Tradicionalmente, el Estado uruguayo determinaba el español como la lengua de identidad nacional y de uso oficial, con esa identidad lingüística pretendía proteger la unidad nacional, porque el portugués era interpretado como amenaza dados los hechos históricos y el contacto geográfico (ELIZAINCÍN, BEHARES, BARRIOS, 1987). Así lo podemos constatar en: "La República Oriental tiene una gran amenaza y un gran peligro para el porvenir, en lo difundido que se hallaba el idioma brasilero en los Departamentos que son limítrofes del Imperio" (VARELA, 2012[1879], p. 124). Sin embargo, con las políticas de globalización e integración, como con los discursos de derechos y diversidad vistos anteriormente, en Uruguay comenzaron a surgir políticas lingüísticas de la educación pública para la valoración positiva de las variedades lingüísticas nacionales ${ }^{3}$ y de la enseñanza del portugués.

Los compromisos supranacionales revelan la forma en que las políticas educativas, que trazan los países, surgen bajo la sombra de lineamientos más generales que provienen de políticas internacionales, como dice Dale (2004, p. 429) "las políticas nacionales son en esencia poco más que interpretaciones de versiones o guiones que son informados por, y reciben su legitimación de, ideologías, valores y culturas de nivel mundial”. Es decir, los procesos de producción de las políticas nacionales resultan de sucesivas recontextualizaciones operadas en cada uno de los contextos de influencia, de producción y de práctica, a partir de las que se crean

\footnotetext{
${ }^{2}$ Artículo 17 - Los idiomas oficiales del Mercado Común serán el español y el portugués y la versión oficial de los documentos de trabajo será la del idioma del país sede de cada reunión. Artículo 24 - Con el objeto de facilitar el avance hacia la conformación del Mercado Común se establecerá una Comisión Parlamentaria Conjunta del MERCOSUR. Los Poderes Ejecutivos de los Estados Partes mantendrán informados a los respectivos Poderes Legislativos sobre la evolución del Mercado Común objeto del presente Tratado. HECHO en la ciudad de Asunción, a los veintiséis días del mes de marzo del año mil novecientos noventa y uno, en un original en los idiomas español y portugués, siendo ambos textos igualmente auténticos. El Gobierno de la República el Paraguay será el depositario del presente Tratado y enviará copia debidamente autenticada del mismo a los Gobiernos de los demás Estados Partes signatarios y adherentes. (Tratado de Asunción, 1991) Disponible en: https://www.parlamentomercosur.org/innovaportal/file/15511/1/tratado_de_asuncion.pdf. Consultado: 10 nov. 2020.

${ }^{3}$ Como explica Díaz (2008 apud TEIXEIRA, 2016a) en una amplia región del nordeste del Uruguay, la lengua materna es una variedad del portugués, estudiada y descripta como "Dialectos Portugueses del Uruguay" (DPU) o "Portugués del Uruguay".
} 
nuevos textos de políticas en un marco de intereses, conflictos, acuerdos y tensiones.

Sin embargo, de lo regional a lo nacional, a pesar de su firma en 1991, Uruguay no cumplió plenamente con su compromiso lingüístico dentro del MERCOSUR, ya que la puesta en acto de la enseñanza de portugués ha sido, en general, lenta y difícil. Fue con el primer gobierno de izquierda (2005-2010) que se inició un proceso de debate nacional que dio lugar a la producción de los textos de una nueva legislación que incluye la educación lingüística.

Vimos en el contexto de influencias las nuevas políticas que redefinen al Estado y que, a su vez, son redefinidas permanentemente por los estados, el Estado adquiere nuevos poderes y ejerce el poder en nuevas formas, en tal sentido, entendemos que "es importante que no subestimemos el poder del Estado, pero también es importante que no sobreestimemos el poder del Estado en abstracto, ni tratemos al Estado como un todo indivisible" (BALL, 2012, p. 7).

\section{SEGUNDO CONTEXTO}

En este contexto de producción del texto político, las políticas alcanzan forma de textos normativos, articulados con el lenguaje del interés público. En la formulación escrita de la política, ocurre discusión, interpretación, reinterpretación y resistencia al interior de complejas relaciones de poder. Desde el enfoque del ciclo de políticas corresponde ver cómo el Estado interviene en la formulación de estas, además de cómo estas se despliegan más allá del control estatal por la acción de otros actores sociales.

Para Ball (1997) es fundamental reconocer que los textos, donde se formulan las políticas y las leyes, no siempre son claros o completos, asimismo, una propuesta de ley será el resultado de compromisos y negociaciones en varias etapas de su creación. Esto ocurre en diversas circunstancias, cuando algunos grupos influyentes impulsan la idea inicial y la negociación de instancias normativas que regularán la aplicación del texto.

De este modo, a partir del contexto de influencia visto en el primer ciclo, en el ámbito nacional de la trayectoria educativa y de las políticas lingüísticas públicas destacamos instancias de producción textual oficial que posibilitaron la puesta en acto de la formación docente de portugués. Esto ocurrió en el segundo gobierno de izquierda ${ }^{4}$, en el período 2005-2010, al desarrollar diversas estrategias de mejora y desarrollo integral del país. Cronológicamente, haremos una breve referencia al Debate Educativo iniciado en 2005 y al Congreso Educativo de 2006, pero analizaremos textos elaborados desde 2006de la Comisión de Políticas Lingüísticas para la Educación Pública (CPLEP) de la ANEP, un artículo específico de la actual Ley General de Educación (LGE), sancionada en 2008, y párrafos significativos del vigente Plan de Estudio del Profesorado de Portugués, también publicado en 2008.

\section{- Debate y Congreso Nacional Educativo}

En 2005, el Ministerio de Educación y Cultura (MEC), la ANEP y la Universidad de la República (UDELAR) convocaron a un Debate Educativo, con múltiples espacios de participación. La Comisión Organizadora del Debate Educativo (CODE) creó 16 Comisiones Departamentales, 18 Comisiones Zonales en Montevideo, 9 en Canelones y 4 en Colonia. También hubo una Convocatoria al Debate Educativo, publicada como llamado general a la ciudadanía. Se organizó dos tipos de participación ciudadana: las Asambleas Territoriales, constituidas por las Comisiones Zonales y Departamentales, y los Encuentros Sectoriales con organizaciones e instituciones, convocadas a nivel local por estas Comisiones y a nivel nacional por la CODE. En las 713 Asambleas Territoriales 19.070 personas participaron en total. 157

\footnotetext{
${ }^{4}$ En toda la historia nacional, solamente existieron tres gobiernos de izquierda, consecutivos de 2005 a 2020.
} 
organizaciones participaron en los Encuentros Sectoriales, a lo que se sumaron asambleas y encuentros en todo el sistema educativo. Al mismo tiempo, por correo electrónico y la página web llegaron 410 documentos, 257 grupales y 153 individuales.

El debate fue la antesala del Congreso Nacional de Educación, realizado en 2006, integrado por 1960 delegados de los colectivos reconocidos previamente por la CODE, democrático en su funcionamiento, gratuito y resolutivo, ya que el Plenario adoptó resoluciones, constando las posiciones minoritarias cuando así fue solicitado por miembros del Congreso. Sus resoluciones constituyen recomendaciones a las autoridades autónomas de la enseñanza y a los poderes Ejecutivo y Legislativo (CODE, 2007).

En nuestro estudio, no dejamos de pensar que, como afirma Foucault (1980), al intentar articular una serie de discursos materializados en recomendaciones o resoluciones institucionales, el análisis de estos se sustenta enlazando el deseo y el poder, los procedimientos que lo controlan y delimitan, así como las condiciones de producción y de utilización. Por lo tanto, si bien el proceso de debate y el congreso realizado generó insumos para la nueva ley, publicados en 2007 por la CODE, constataremos, más adelante, de qué modo o en qué medida la LGE $\mathrm{N}^{\circ} 18.437$, sancionada en 2008, contempla estos discursos.

\section{Este Congreso solicita que la Ley de Educación:}

- contemple en su articulado la importancia de la multiculturalidad y del plurilingüismo.

- mencione el derecho de todo ciudadano al acceso al estudio de múltiples culturas y lenguas, que no exista una lengua hegemónica en el currículo del sistema educativo. - prevea la formación inicial y continua de calidad de los docentes de lenguas extranjeras en todos los niveles, siendo asegurada por el sistema educativo estatal.

\section{Enseñanza del portugués y el inglés}

Uruguay está inmerso en el MERCOSUR y se considera que como miembro fundador del mismo se enseñe portugués con carácter obligatorio en todo el territorio nacional desde el nivel inicial. Asimismo, se considera al idioma inglés esencial para enfrentar las coyunturas del siglo XXI. Se mociona para que ambas lenguas sean incorporadas a los respectivos diseños y programas (CODE, 2007, p. 296).

Un proyecto educativo lingüístico deberá contemplar íntegramente el Sistema Nacional de Educación asegurando la coordinación y articulación entre los distintos subsistemas de la ANEP y Educación Terciaria. Un proyecto educativo debe estar respaldado por la existencia de una matriz docente adecuada. Se propone que el IPA agregue la carrera de Portugués (CODE, 2007, p. 239).

\section{- Comisión de Políticas Lingüísticas para la Educación Pública}

En esta etapa de la trayectoria de la política educativa uruguaya, entre las discusiones y producciones de documentos e informes técnicos de 2006 a 2007, la ANEP creó la Comisión de Políticas Lingüísticas para la Educación Pública (CPLEP) para aportar a la Ley No 18.437. Si bien citamos textos políticos producidos para dar cuenta del proceso de materialización de los discursos que confluyeron después de discusiones de distintos agentes de la educación y de especialistas en investigación lingüística, destacamos los vinculados directamente con portugués y la formación docente, a saber: Marco Orgánico de Referencia de las Políticas Lingüísticas en la Educación Pública; Propuesta de Reestructura de los Componentes Curriculares y Extracurriculares del Dominio Lingüístico de los tres Subsistemas; Recomendaciones referidas a la Formación Docente para el Dominio Lingüístico; Distribución actual del Portugués del Uruguay en territorio uruguayo; Datos sobre el dominio lingüístico en el nivel superior de educación; Panorama de las normas jurídicas uruguayas con consecuencias 
político-lingüísticas.

Veamos algunos ejemplos de conceptualizaciones, objetivos, recomendaciones que movilizan discursos más recientes sobre las lenguas y su enseñanza en los espacios públicos, que están en concordancia con las políticas manifestadas en el Debate Educativo y publicadas por la CODE. Asimismo, las designaciones de las lenguas como maternas, primeras, segundas, extranjeras, regionales, vecinas, originarias, de herencia o de origen, entre otras, más allá de las conceptualizaciones teóricas y de usos oficiales en documentos, evidenciamos las relaciones de imaginario-memoria y sujeto-lengua.

Las segundas lenguas y las lenguas extranjeras constituyen un componente fundamental en la formación del individuo, en virtud de sus potenciales papeles en la ampliación del acceso a la información, de la participación social y cultural y de su contribución al desarrollo cognitivo. La oferta plurilingüe, con las modalidades y flexibilidades que cada subsistema permita, es la que se considera deseable, tomando en cuenta la variedad de situaciones, regiones, grupos humanos e intereses (CPLEP, 2006-2007, Documento 1, 5. Principios rectores 7., p. 26).

Nos referimos aquí al hecho de que la introducción de una LE con una metodología apropiada genera y estimula el entendimiento intercultural, la valoración de las culturas propia, vecina y ajena. Es así que el espacio de enseñanza de lenguas es considerado actualmente como un ámbito privilegiado para el trabajo en valores positivos como la diversidad cultural y la aceptación de las diferencias entre los distintos grupos humanos (CPLEP, 2006-2007, Documento 2, 3.4. Justificación de esta propuesta, p. 51).

En cuanto al Portugués, el enclave regional del Uruguay constituye una motivación para la inclusión de esa lengua, que se relaciona con la necesaria comunicación a nivel regional así como en los acuerdos firmados por Uruguay y Brasil en materia de lengua (Tratado de Asunción). Más allá del contexto geográfico y las decisiones políticas, el Portugués constituye, además, una lengua que alberga una extensa producción literaria. A estas razones de tipo instrumental y cultural, se debe agregar la consideración del estatus especial que tiene el Portugués en el Uruguay, debido a su presencia histórica en nuestro territorio, de modo que su variedad uruguaya (llamada por los investigadores "Dialectos Portugueses del Uruguay") resulta estar muy vinculada a la lengua materna de una parte de la población del país, como se tratará más adelante en este Documento (Capítulo 5). Es decir, la inclusión obligatoria de la enseñanza del portugués tiene una justificación de tipo "externa" (comunicación con los vecinos de un país limítrofe y el único país latinoamericano con una lengua oficial distinta del español), pero también una de tipo "interna" con potenciales valores de integración de la sociedad uruguaya (CPLEP, 2006-2007, Documento 2, 3.4. Justificación de esta propuesta, p. 53).

La enseñanza del portugués está justificada por razones políticas y económicas de orden regional, además de otras cuestiones etnolingüísticas propias del país. Vemos, como explica Barrios (2011), que la selección de las lenguas se hace por argumentos de orden instrumental, cognitivo e identitario. También percibimos la redundancia de sentidos de la interculturalidad y del respeto a la diversidad. Según Barrios (2011), en general, las propuestas políticolingüísticas tienen un gran impacto y buscan influenciar profundamente los modelos de identidad. De este modo, la elección del portugués como segunda lengua obligatoria en la educación pública para todo el país, supone un cambio de modelos etnolingüísticos, con consecuencias particulares según la región: en la frontera con el Brasil, la consolidación y la valorización de la tradición brasileña; en el resto del país, la reformulación de modelos tradicionales fuertemente ligados a la inmigración.

Evidenciamos una consecuencia de los documentos firmados, es decir, se procura avanzar en la concreción de los acuerdos regionales del MERCOSUR. Notamos un efecto 
cascada, o dominó, puesto que textos de otros organismos y textos de la CPLEP reproducen y reformulan discursos de diferentes organismos trasnacionales y nacionales. No obstante, sabemos que los discursos encuentran dificultades en la puesta en acto institucional para el cumplimiento de propuestas oficiales.

De este modo, como explica Ball (1993), conceptualizar la política como texto implica entenderla como intervenciones textuales, como codificaciones multifacéticas producidas a través de compromisos, luchas, interpretaciones y reinterpretaciones públicas autorizadas. Por consiguiente, la adhesión, modificación y definición de los textos oficiales constan, en primera instancia, sólo en términos discursivos, puesto que la definición de instrumentos, programas, y lineamientos de acción podrá realizarse con mucha posterioridad, porque de hecho todavía no se ha incluido la enseñanza obligatoria de portugués.

A partir de los textos de la CPLEP enfatizamos que las políticas lingüísticas, como enunciados y parte del archivo (FOUCAULT, 1980), pueden ser comprendidas como gestos de poder del Estado que serán ejercidas y efectuadas continuamente en el interior de la sociedad, a través de diversos dispositivos y prácticas que constituyen una gran red, forman parte de un sistema de saberes y conocimientos directamente aplicable a la población en forma de técnicas de gubernamentalidad (FOUCAULT, 1979).

Por lo tanto, podemos analizar esos discursos "bajo un grupo de explicaciones que ligan las instituciones, los procesos económicos y sociales, las formas de comportamientos, los sistemas de normas, las técnicas y tipos de clasificaciones" (BERT, 2013, p.182). Siendo así, las acciones de políticas lingüísticas precisan aplicarse como saber comprendido, como práctica, acontecimiento y materialidad, son elementos de un dispositivo político que se articula con la estructura económica, porque, según Foucault (1980), todo saber tiene su origen en las relaciones de poder.

En los textos de la CPLEP hay movimientos en doble sentido, nacional-regional, que funcionan tanto para justificar la enseñanza del portugués como para discutir aún más sobre todos los aspectos involucrados en las políticas del Estado. Y entre esos movimientos, nuevamente, surge la frontera que constituye un espacio significativo para las decisiones políticas lingüísticas y educativas.

\footnotetext{
En lo que respecta a Educación Media, según se desprende del relevamiento hecho de los Planes de Estudio de nuestros sistemas educativos, las lenguas extranjeras han estado siempre presentes, aunque en diferente medida, en los programas. Éstas han sido siempre las mismas (Francés, Italiano, Alemán) en los diferentes planes hasta 1996, momento a partir del cual se incorpora Portugués en una modalidad optativa y no obligatoria, como respuesta a compromisos asumidos al integrarnos al Mercosur (Tratado de Asunción). (CPLEP, 2006-2007, Documento 2, 3.2. El estado de situación de la enseñanza de lenguas extranjeras en los tres subsistemas, p. 47).
}

Este texto trae las memorias del estatus de la historia del portugués en la educación pública, como también en los discursos de los sujetos en la sociedad, sea por reformulación o por oposición. La referencia histórica que funciona en el proceso discursivo como antecedente está exteriorizada como compromisos asumidos en el MERCOSUR. Además, vemos en la historia de la enseñanza uruguaya el privilegio de lenguas europeas. Por eso, es importante notar que en los textos predomina un interdiscurso internacional similar al materializado en los acuerdos de la Unión Europea, el Marco Común Europeo de Referencia para las Lenguas y la Carta Europea sobre las Lenguas Minoritarias o Regionales, que también tiene efectos en los documentos recientes. No obstante, para Teixeira (2016b), en la frontera muchos hablantes sufren represión y no pueden usar su lengua materna DPU en las escuelas, sin protección contra la discriminación según artículos 7 y 19 de los Derechos Lingüísticos (1996). 


\title{
- Ley General de Educación
}

A partir de textos políticos del Debate Educativo y del primer Congreso Nacional de Educación, venimos exponiendo que la incorporación de nuevas normativas define metas, posibilidades y limitaciones de las distintas instituciones y agentes educativos a través de la legitimación de ciertos modelos de funcionamiento macro y micro institucional, dentro de lo que Ball denomina micropolíticas. Por lo tanto, la nueva Ley General de Educación $\mathrm{N}^{\mathrm{o}}$ 18.437, de 2008, reconfigura relaciones y realidades dentro del sistema educativo uruguayo. Veamos los postulados lingüísticos:

\begin{abstract}
La educación lingüística tendrá como propósito el desarrollo de las competencias comunicativas de las personas, el dominio de la lengua escrita, el respeto de las variedades lingüísticas, la reflexión sobre la lengua, la consideración de las diferentes lenguas maternas existentes en el país (español del Uruguay, portugués del Uruguay, lengua de señas uruguaya) y la formación plurilingüe a través de la enseñanza de segundas lenguas y lenguas extranjeras (Parlamento del Uruguay, LGE 2009, Tit. II, Cap. VII, Líneas transversales, Art. 40, E, 5, p. 7).
\end{abstract}

Después de más de un siglo del documento legal anterior, vigente hasta la firma de la LGE, como antítesis, se incorpora una serie de planteamientos nuevos para las lenguas minoritarias locales, segundas y extranjeras. Sin embargo, no se textualiza portugués como lengua plena, tal como ocurre en los documentos del CODE (2007) y del CPLEP (2006-2007), pareciera que en la designación aún hubiera vestigios de la tradicional política lingüística que obstaculizaba la expansión del portugués en territorio uruguayo.

En los proceso históricos nacionales encontramos un pasado en el cual, como afirma Barrios (1996), la política lingüística buscaba eliminar las lenguas minoritarias del país. A fines del siglo XIX, para alcanzar la unificación lingüística, el Estado aplicó la Ley de Educación Común $N^{\circ} 1350$ de 1877 que impuso la educación primaria como obligatoria y el español como lengua de enseñanza sobre todo el territorio nacional. Esa ley fue aplicada en todo el país, independientemente de la diversidad sociolingüística que habitaba aquel espacio y ello tuvo consecuencias sobre resultados escolares que, como afirma Behares (1984), se manifestaron disímiles e inesperados según las diferentes regiones del país. Uno de los aspectos de las condiciones de producción del documento anterior refiere a que J. P. Varela, su promotor, tenía la convicción de que en el norte del país la lengua nacional estaba ya casi perdida, debido a que el portugués era hablado por una gran mayoría de la población. Para resolver ese problema de la frontera, tal como se lo consideraba, el español fue valorizado y el portugués denigrado (ELIZAINCÍN, BEHARES, BARRIOS, 1987).

En la nueva ley, como el único artículo referido a la educación lingüística, aparece en las denominadas Líneas transversales, podríamos pensar que no tiene un protagonismo relevante en la materialidad del documento. No obstante, como se trata de una ley, comprendemos que su valor socio-político es muy significativo, además, identificamos las influencias políticas de documentos internacionales, regionales y nacionales, como demostramos en este estudio. Asimismo, la hegemonía entre lenguas envuelve estigmas y prejuicios según el status (CALVET, 1996, p. 11 apud TEIXEIRA, 2013, p. 2) atribuido a cada variedad en la educación, política, economía y sociedad en general.

\section{- Plan de Estudios Profesorado Portugués}

A partir de la adhesión del CFE a las Recomendaciones de la CPLEP, los entes de concertación política tienen la necesidad de alinearse con estas nuevas propuestas de políticas lingüísticas. Hay un sistema, es decir, coordinación, implementación y ejecución de las políticas 
públicas que desarrollan los organismos públicos sobre la políticas educativas actuales.

Este documento constituye una materialidad que ocurre en la dimensión textual del discurso y, a su vez, como ideología concreta que se realiza, orienta las prácticas de la formación docente. El plan de estudio es un efecto de los documentos nacionales producidos en este ciclo político. Este documento, como instancia previa a la puesta en acto de los cursos de la carrera en la ANEP, constituye la base discursiva en la que se apoya el contexto de la práctica política.

Para Ball los textos de las políticas constituyen el discurso oficial del Estado que busca constituir y cambiar las prácticas educativas (LINGARD \& OZGA, 2007). Así, se presenta a la política como una codificación compleja de significados que se inscriben en textos de la política (tales como leyes, resoluciones, memorandos, y documentos) que son a su vez objeto de diversas y complejas decodificaciones y re codificaciones. Como este abordaje asume que no se sabe de antemano si los actores van a apropiarse o no de las políticas ni cuáles serían los efectos, un estudio de campo podría dar indicios sobre las puestas en acto del profesorado de portugués en los institutos docentes de nuestro país.

Los planes de estudios fueron hechos con la participación de profesores del DELEX en conjunto con el CFE. Eso demuestra un desplazamiento interno de las responsabilidades y de las posibles autorías en los textos políticos. Reconocemos que cuando los profesores tienen un papel definido en el proceso de elaboración de un plan de estudio se sienten más motivados y comprometidos para que este resulte exitoso.

El portugués es, para el Uruguay, una lengua propia, vecina y ajena al mismo tiempo. Propia, en tanto existe en Uruguay población hablante de portugués, cuya presencia se explica por razones históricas. En una amplia región del noreste del Uruguay existe población cuya lengua materna es una variedad del portugués; esta variedad lingüística, ha sido estudiada y descrita en trabajos de investigación y ha sido denominada "Dialectos Portugueses del Uruguay" (DPU) o "Portugués del Uruguay". El portugués es también una lengua vecina en lo que se refiere al enclave regional del Uruguay en relación a Brasil. Esta relación de vecindad territorial tiene consecuencias en el ámbito subjetivo de la percepción que los hispano-hablantes uruguayos tienen del portugués, así como en aspectos públicos vinculados a los acuerdos de integración políticos y comerciales. Por último, puede decirse también que el portugués es una lengua ajena, en tanto para muchos uruguayos se trata de una lengua desconocida, con la cual han tenido escaso o nulo contacto, con relativamente poca presencia en los medios de comunicación y con limitada inteligibilidad. Dada esta variedad de situaciones, puede afirmarse que el portugués no es, para nuestro país, una lengua extranjera en el mismo sentido que otras, ya que razones históricas y de territorialidad definen una vinculación especial entre el Uruguay y la lengua portuguesa (CFE, DELEX, Plan de Estudios 2008, Profesorado de Portugués, Fundamentación, p. 1).

En lo que se refiere a los aspectos culturales vinculados a la enseñanza de una lengua, el profesor de portugués como segunda lengua o lengua extranjera, debería acercar al estudiante a la diversidad cultural vehiculizada por el portugués en Brasil y en los demás países de lengua portuguesa del mundo. Por este motivo, necesita disponer de un conocimiento profundo y amplio de la lengua y sus variedades, incluyendo aquellas habladas en áreas fronterizas de Uruguay, que le permita informar a sus alumnos sobre la presencia de portugués en el territorio nacional y promover una revalorización de la cultura lusófona uruguaya (CFE, DELEX, Plan de Estudios 2008, Profesorado de Portugués, Acerca del perfil de egreso, p. 3-4).

A partir de la designación lengua propia pensamos en los sujetos constituidos en portugués, como lengua oficial y lengua nacional, por ejemplo. Entendemos que lengua vecina refiere al espacio geográfico, al espacio discursivo y a la familia de lengua. La lengua ajena parece expresar desconocimiento, negación o indiferencia hacia el portugués y/o Brasil. Nuevamente se manifiestan razones históricas como condiciones de producción en las luchas entre español y portugués ocurridas en el actual territorio uruguayo. 
En general, el MERCOSUR configura un nuevo y un viejo espacio enunciativo. Nuevo, porque según Guimarães (1997), las políticas, en relación con las lenguas, constituyen nuevos espacios para la circulación del portugués y el español, principalmente. Pero para él, eso no es suficiente; para que de hecho haya una integración es necesario que se afirme el derecho de no hablarse la misma lengua de todos. Configura un viejo espacio enunciativo, porque, a través de los discursos materializados en la textualidad de los documentos referentes al MERCOSUR, se constata la reproducción de los discursos homogeneizadores y hegemónicos que silencian la diversidad en nombre de una falsa uniformidad.

En la primera cita notamos un discurso compartido con la CPLEP discutida más arriba. Nuevamente, se enfatiza que en Uruguay las lenguas español y portugués mantienen una historia de contacto de sustrato y adstrato, por las épocas de conquistas europeas en América, así como una relación horizontal, por las disposiciones geográficas de las políticas territoriales. De este modo, los sujetos habitantes de diferentes partes del país mantienen relaciones diversas con el portugués, según signifique para cada hablante uruguayo LM, L2 o LE. En el caso de LM, sabemos que en la historia del sujeto, constituye su relación con lo real y su subjetividad, por lo tanto, el Estado debe contemplar Derechos Lingüísticos de la UNESCO.

Evidenciamos cómo del plan de estudios responde a las recomendaciones de la CPLEP. Igualmente, reconocemos el contexto de influencia en relación con los demás discursos y documentos que componen nuestro corpus representativo. Confirmarnos también que en estos casos, los aspectos públicos pertenecientes al Estado procuran alcanzar y/o mantener una linealidad coherente en las secuencias de documentos, pero que discursivamente pueden generar ciertas ambigüedades o vacíos legales.

Como en otros textos analizados, las metonimias de integración generan sentidos de asociación y de cooperación entre las instituciones y los mercados, pero no necesariamente entre las personas. Asimismo, estas relaciones de grupo no presuponen una equivalencia de poderes (FOUCAULT, 2000) para los asociados en los acuerdos de integración política y comercial. A la par, encontramos silencios textuales de la integración educativa, social, cultural.

Al igual que en los otros textos políticos, las condiciones sociohistóricas son movilizadas como fundamentos. La vecindad parece tener sentidos predominantes de fuerzas políticas y económicas. Por tal razón, podríamos cuestionar si la vecindad es solamente territorial o es también por los sentidos de afinidades culturales vistos en ciertos discursos como comunes, por la historia compartida. Según sea la interpretación al respecto, habría un silencio en los aspectos culturales, al igual que en otras expresiones analizadas.

\section{TERCER CONTEXTO}

El contexto de la práctica está ligado al proceso de traducción que los docentes, actores centrales de la práctica educativa, hacen del texto político con el propósito de dotarlo de sentido, ya que el modo en que piensan tiene profundas implicaciones en su puesta en acto. Por lo tanto, las políticas no son simplemente implementadas en el contexto de práctica, sino que están sujetas a interpretación y recreación (BOWE et al., 1992 apud MAINARDES, 2006).

Ball $(1998,2001,2004)$ resalta la importancia de cada institución educativa para el desarrollo de procesos de interpretación y traducción de las políticas, con las marcas identificativas de cada una. La "interpretación está comprometida con el lenguaje de la política, mientras que la traducción se relaciona al lenguaje de la práctica. La traducción es una suerte de tercer espacio entre política y práctica" (BALL et al., 2011, p. 45). Por ende, interpretación tiene que ver con la lectura inicial de las políticas como decodificación, pero traducción es un proceso que implica la producción de nuevos textos institucionales y su puesta en acto.

La instalación de una normativa es, principalmente, una acción política, fundamentada 
solo en parte en argumentos académicos, como por ejemplo, la generación de nuevos marcos prescriptivos y curriculares, como ocurrió en 2008 con el plan de estudio del profesorado portugués. Como vimos en el apartado anterior, en el marco de las nuevas normativas del Estado, dicho texto político da cuenta de los sentidos asignados al portugués como efectos de una red de políticas públicas. En atención a la diversidad lingüística la CPLEP propone la obligatoriedad de la enseñanza del portugués en los niveles primarios y secundarios, para ello es imprescindible contar con profesores egresados de la formación docente de la ANEP.

De este modo, en el tercer ciclo político, las respuestas a los textos tienen consecuencias reales, vividas en el contexto de la práctica. Sin embargo, en esta oportunidad no podremos dar cuenta de ello, porque requiere recoger, sistematizar y analizar los sentidos otorgados por los actores institucionales involucrados en la puesta en acto de la política educativa de la formación docente de portugués Uruguay. No obstante, a modo de referencia general, podemos informar que el profesorado de portugués fue puesto en práctica, gracias a los ciclos políticos anteriores con producción de documentos oficiales, a partir del 2009. Las políticas establecidas permitieron distintos contextos de práctica, en ese entonces la carrera fue implementada en el Instituto de Profesores Artigas (IPA), en Montevideo, la capital ubicada en la región sur, y en el Centro Regional de Profesores de Rivera (CeRP del Norte), región norte. Después, en 2015, pasó a realizarse en el IPA y en la modalidad semipresencial y, a partir de 2020, a existir únicamente como profesorado semipresencial.

\section{CONSIDERACIONES FINALES}

En nuestro escenario discutimos políticas caracterizadas como redes, consecuentes de la conjunción de influencias e interdependencias (BALL, 2001), verificadas a partir del primer contexto del ciclo de políticas. Comprobamos que las políticas nacionales resultan de la combinatoria de lo global, lo regional y lo local. En tal sentido, constatamos que la respuesta del Estado a las necesidades sociales concedió una importante reconfiguración a las políticas educativas uruguayas.

El análisis realizado nos permitió obtener indicios para comprender lo que sucede en el contexto de producción de políticas, las que mueven y cambian sus significados en las arenas de la política educativa, cambian las representaciones y los intérpretes claves. Estas transformaciones impulsaron, entre otras instancias de la ANEP, la confección de nuevos planes oficiales para la formación docente en Uruguay, en la cual surge, por primera vez en la historia educativa, la puesta en acto de la formación docente de portugués.

Las políticas son especialmente relevantes cuando se arraigan en instituciones de alta legitimidad social, directamente conectadas con la toma de decisiones o cuando son asumidas y promovidas por actores particularmente influyentes. En nuestro estudio, pudimos evidenciar como el Estado uruguayo generó un proceso histórico y democrático de gran participación ciudadana en las políticas públicas, iniciado con el Debate Educativo, seguido del primer Congreso Nacional Educativo para llegar al poder ejecutivo del parlamento nacional y sancionar la Ley General de Educación.

Dichos acontecimientos participativos y legales constituyeron respuestas contundentes del Estado a los contextos de influencia, en los cuales destacamos las políticas educativas manifestadas en los Derechos Lingüísticos y el MERCOSUR. Asimismo, en el contexto nacional, las comisiones de autoridades y especialistas fueron claves al alinearse a los discursos internacionales y regionales de diversidad lingüística para dar lugar a los textos políticos de la ANEP, como los de la CPLEP y el fundamental plan de estudio del profesorado de portugués que cambiaron las políticas lingüísticas nacionales. De este modo, la política educativa uruguaya reflejó en diversos documentos sus iniciativas para cumplir con compromisos 
lingüísticos internacionales, aunque en la práctica persistan incongruencias.

En concordancia, Ball (2002) afirma que los textos son producto de compromisos en la influencia inicial, en las micro políticas de la formulación legislativa, en el proceso parlamentario y en las políticas y micro políticas de los grupos de interés. Por consiguiente, evidenciamos que, en términos de discursos oficiales y ejecución de políticas uruguayas, el Estado Nacional buscó dar nuevos sentidos a diversas dimensiones, como las políticas educativas, las propuestas de transformación y la mejora de la educación que requirieron el compromiso político de distintos sectores sociales que interactuaron con el sistema educativo.

Como detallamos a lo largo de este estudio, la formulación de las actuales políticas lingüísticas supuso un proceso complejo de interacciones entre diferentes agentes con desencuentros, acuerdos y negociaciones que finalmente permitió la creación del plan de estudio para la formación docente de portugués de la ANEP. De este modo, entendemos, como expresa Ball (1997), que las políticas, como el plan de estudio, no dicen que hacer, sino crean las circunstancias, pero los actores crean respuestas y las construyen en contexto. En este sentido, a partir de la nueva normativa que cobró relevancia en la agenda del Estado, en otra oportunidad, podríamos continuar con una investigación de campo para revelar, en el contexto de la práctica, aspectos del contexto de los efectos y estrategias políticas de la aún vigente formación de profesores de portugués.

\section{REFERENCIA}

ANEP. Administración Nacional de Educación Pública. Documentos de la Comisión de Políticas Lingüísticas en la Educación Pública. Montevideo: ANEP, 2008.

AVELAR, Marina. Entrevista a Stephen J. Ball: Analizando su contribución a la investigación de políticas educativas. Archivos de análisis de políticas educativas, v. 24, p. 24, feb. 2016.

BALL, Stephen J. Textos, discursos y trayectorias de la política: la teoría estratégica. Páginas. Revista de la Escuela de Ciencias de la Educación, n. 2 y 3, 2016.

BALL, Stephen J. Global education inc: Nuevas redes políticas y el imaginario neoliberal. Routledge, 2012.

BALL, Stephen J.; MAGUIRE, Meg; BRAUN, Annette. How schools do policy: Policy enactments in secondary schools. Londres: Routledge, 2011.

BALL, Stephen J. Diretrizes políticas globais e relações políticas locais em educação. Currículo sem fronteiras, v. 1, n. 2, p. 99-116, 2001.

BALL, Stephen J. Big policies/small world: An introduction to international perspectives in education policy. Comparative education, v. 34, n. 2, p. 119-130, 1998.

BALL, Stephen J. Reforma educativa: un enfoque crítico y post- estructural. Buckingham: Open University Pressm, 1997.

BALL, Stephen J. ¿Qué es la política? Textos, trayectorias y cajas de herramientas. The Australian Journal of Education Studies, v. 13, n. 2, p. 10-17 de 1993.

BARRIOS, Graciela. El tratamiento de la diversidad lingüística en la educación uruguaya (2006-2008). Letras, v. 21, n. 42, p. 15-44, 2011. 
BARRIOS, Graciela. Planificación lingüística e integración regional: el Uruguay y la zona de frontera. In: MENINE TRINDADE, Aldema y BEHARES Luis. E. (Org.) Fronteiras, Educação, Integração. Santa María, Pallotti, 1996. 83-110.

BEECH, Jason; MEO, Analía Inés. Explorando el uso de las herramientas teóricas de Stephen J. Ball en el estudio de las políticas educativas en América Latina. Education Policy Analysis Archives/Archivos Analíticos de Políticas Educativas, v. 24, p. 1-19, 2016.

BEHARES, Luis E. Planificación lingüística y educación en la frontera uruguaya con Brasil. Montevideo: Instituto Interamericano del Niño, 1984.

BERT, Jean-François. Pensar com Michel Foucault. São Paulo: Parábola, 2013.

BOWE, Richard; BALL, Stephen J.; GOLD, Anne. Reformar la educación y cambiar las escuelas: estudios de caso en sociología de políticas. London: Routledge, 1992.

CMCE. Comité de Ministros del Consejo de Europa. Carta Europea de las lenguas regionales y minoritarias. Estrasburgo, 5 de noviembre de 1992. Disponible en: http://www.consello.org/pdf/cartaeuropea92.pdf. Consultado: 20 oct. 2020.

CODE. Comisión Organizadora del Debate Educativo. Informe Final. Montevideo: MEC, 2007. Disponible en: https://docplayer.es/348712-Comision-organizadora-del-debateeducativo-code-informe-final.html. Consultado: 01 nov. 2020.

CONSEJO DE EUROPA. Marco Común Europeo de Referencia para las Lenguas. 2001. Disponible en: https://cvc.cervantes.es/ensenanza/biblioteca_ele/marco/cvc_mer.pdf. Consultado: 05 oct. 2020.

DALE, Roger. Globalização e educação: demonstrando a existência de uma" cultura educacional mundial comum" ou localizando uma "agenda globalmente estruturada para a educação"? Educação \& sociedade, v. 25, n. 87, p. 423-460, 2004.

ELIZAINCÍN, Adolfo; BEHARES, Luis Ernesto; BARRIOS, Graciela. Nos falemo brasilero: dialectos portugueses en Uruguay. Montevideo: Amesur, 1987.

FOUCAULT, Michel. Vigilar y castigar: nacimiento de la prisión. Siglo xxi, 2000.

FOUCAULT, Michel. Microfísica del poder. Madrid: Ediciones La Piqueta Seseña, 1980.

FOUCAULT, Michel. Ideology and consciousness. Governmentality, 1979, v. 6, p. 5-21.

GUIMARAES, Eduardo. Política de línguas na América Latina. In: POLÍTICAS LINGÜÍSTICAS PARA AMÉRICA LATINA, 1997, Buenos Aires. Actas del Congreso Internacional. Buenos Aires: Universidade de Buenos Aires, 1999. p. 297-304.

LINGARD, B.; OZGA, J. Introduction: reading education policy and politics. In: The Routledge Falmer reader in education policy and politics. London: Routledge, 2007.p.1118.

MAINARDES, Jefferson. Abordagem do ciclo de políticas: uma contribuição para a análise de políticas educacionais. Educação \& Sociedade, v. 27, n. 94, p. 47-69, 2006. 
MIRANDA, Estela. Una "caja de herramientas" para el análisis de la trayectoria de la política educativa. La perspectiva de los ciclos de la política (Policy Cycle Approach). In MIRANDA, Estela y PACIULLI, Newton (Ed.) Pensar la Educación Pública. Aporte desde Argentina y Brasil. Córdoba, Argentina: Editorial FFYH-UNC. Córdoba. FFyH. UN Córdoba, 2011. p. 105-126.

RME. Reunión de Ministros de Educación del MERCOSUR. Acta 1/93, art 8. Asunción, 30 de junio de 1993. Disponible en: http://edu.mercosur.int/esES/component/jdownloads/viewcategory/295-1993.html. Consultado: 10 nov. 2020.

ROBERTSON, Roland. Glocalización: tiempo-espacio y homogeneidad-heterogeneidad. In: MONEDERO, Juan Carlos. Cansancio del Leviatán: problemas políticos de la mundialización. Trotta, 2003. p. 261-284.

SEM. Sector Educativo del MERCOSUR. Plan Estratégico 2006-2010. 2006. Disponible en: https://www.museofigari.gub.uy/innovaportal/file/1681/1/plan_sem_2006-2010_anexoiv.pdf. Consultado: 5 nov. 2020.

TEIXEIRA, Edilson. Enseñanza pública del portugués en Uruguay. abehache. Revista da Associação Brasileira de Hispanistas, v. 1, n. 10, p. 100-128, 2016a. Disponible en: https://revistaabehache.com/ojs/index.php/abehache/article/view/8/8. Consultado: 11 nov. 2020 .

TEIXEIRA, Edilson. Langues en contact: le cas du portugais et du portugnol dans l'histoire et l'éducation de l'Uruguay. In: GARIN, Virginia et DJORDJEVIC LEONARD (Comp.) Contacts (ou conflits) de langues en contexte postcommuniste et postcolonial. Presses Universitaires de la Méditerranée, Montpellie. 2016b. p. 203-225. Disponible en: http://repositorio.cfe.edu.uy/bitstream/handle/123456789/1037/Teixeira\%2cE.Langues.pdf?se quence $=2 \&$ isAllowed $=y_{.}$. Consultado: 10 nov. 2020 .

TEIXEIRA, Edilson. Formación docente de portugués en Uruguay: Nociones de política y planificación lingüística. In: IPA. Anales del Instituto de Profesores Artigas. ANEP. Segunda Época. Tomo VI. Montevideo. 2013, p. 373-383. Disponible en: http://repositorio.cfe.edu.uy/bitstream/handle/123456789/1058/Teixeira\%2cE.\%2c\%20Forma cion.pdf? sequence=2\&isAllowed=y. Consultado: 11 nov. 2020 .

UNESCO. Organización de las Naciones Unidas para la Educación, la Ciencia y la Cultura. Declaración Universal de los Derechos Lingüísticos. Barcelona de 6 a 9 de Junio de 1996. Disponible en: https://www.pencatala.cat/wp-content/uploads/2016/02/dlr_espanyol.pdf. Consultado: 10 oct. 2020.

URUGUAY. Ley $n^{\circ}$ 18.437, de 12 de diciembre de 2008. Ley General de Educación. Disponible en: https://www.impo.com.uy/bases/leyes/18437-2008. Consultado: 10 nov. 2020.

URUGUAY. Ley $n^{\circ} 1350$, de 24 de agosto 1877. Instrucción pública. Enseñanza primaria. Educación Común. Disponible en: https://legislativo.parlamento.gub.uy/temporales/S2017070463-004723454.pdf. Consultado: 11 nov. 2020.

VARELA, José Pedro. Memoria del Inspector Nacional. 1877-1878. In. A. Palomeque (comp.), José Pedro Varela y su tiempo. Escritos de José Pedro Varela y documentos de época. v. 6. Montevideo: ANEP, 2012. p. 89-252. 